Postgrad. med. J. (1966), 42, 548.

\title{
THE INTERNAL APPROACH FOR INGUINAL HERNIAE
}

\author{
BRIAN V. McEvedy, M.Ch., F.R.C.S. \\ The Royal Victoria Infirmary, Newcastle-upon-Tyne
}

WHILE recurrences occur after inguinal hernia operations no-one can be completely satisfied with the operations practised. During the last 50 years repeated attempts have been made to introduce an internal approach for the repair of inguinal herniae, but these have never proved popular and the inguinal canal incision remains the routine operation; possibly the time has now arrived for a reappraisal of the position.

In 1919 La Roque in America had described an approach through the routine inguinal incision, the extraperitoneal space being entered through a small muscle-splitting incision about an inch above the internal ring. By 1924 he had operated on 500 cases, and in 1932 reported on 1,700 cases, with a very low recurrence rate.

His operation never seems to have become popular despite a plea for it by Williams (1938).

In England Sir Leanthal Cheatle was the first to describe the extraperitoneal approach (1920). He used a vertical rectus-splitting incision, but later (1921) preferred a Pfannenstiel; he mobilised and isolated the processus vaginalis from the vas and vessels and then divided it; the distal end was either excised or ligated as low as possible. He carried out no repair and ended by examining the opposite side through the same incision; no follow-up of his series was ever published.

Henry (1936a) redescribed Cheatle's approach, via a midline incision, at first recommending it for femoral and then for inguinal herniae (1936b). In the inguinal case he repaired the internal ring by suturing the edges of the transversalis fascia firmly round the cord.

The extraperitoneal approach and midline incision were also advocated by Jennings and his associates (1942). However, Jennings later returned to the orthodox method as he found that the inability to repair anything other than the transversalis fascia was unsatisfactory in large indirect herniae (Jennings 1960).

\section{The Internal Approach: Extraperitoneal}

The use of the Rectus Sheath approach for femoral herniae by Peter G. McEvedy (1950) re-awakened interest in this approach to inguinal herniae either through an oblique incision (McEvedy 1958) or through a transverse incision three inches above the inguinal ligament (Nyhus, Condon and Harkins, 1960; Smith, 1962). However, it seems that while there are certain advantages in the extraperitoneal approach, the disadvantages preclude its routine use.

The main advantages are:-

1. The indirect sac is easily isolated without risk of damage to the vas, vessels or bladder; the incision is above the peritoneal thickening at the internal ring which often makes the orthodox approach tedious and incomplete and there is no risk of leaving a small diverticulum of peritoneum projecting inte the internal ring.

2. By palpation and under direct view, othe herniae on the same side can be discovered an through some approaches the opposite side can als be investigated.

3. In obstructed or strangulated herniae the bowel is under direct vision at the neck and resection is easier.

The disadvantages are:-

1. The internal repair is often difficult and sometimes unsatisfactory or incomplete; this is particularly true in the case of the direct inguinal hernia.

2. In the indirect hernia the use of the transversalis fascia to narrow the internal ring can lead to difficulty in placing the sutures as the condensation of fascia overlying the inguinal ligament is rather indefinite; however, it is probably more likely to hold sutures and to unite satisfactorily than the unphysiological suturing of internal oblique muscle to the inguinal ligament.

3. In the midline or lateral rectus sheath approaches any repair in the inguinal canal is impossible and while undoubtedly a sound repair on the inner aspect is better than a sound repair of external oblique, in many cases the external oblique and the external ring are stretched and repair of these structures is indicated.

4. In the large indirect sac, the whole sac cannot be excised, while in some cases of strangulation or incarceration it may be impossible to deal with adhesions in the sac or narrowing at the external ring. 
Summary of Extraperitoneal Approaches

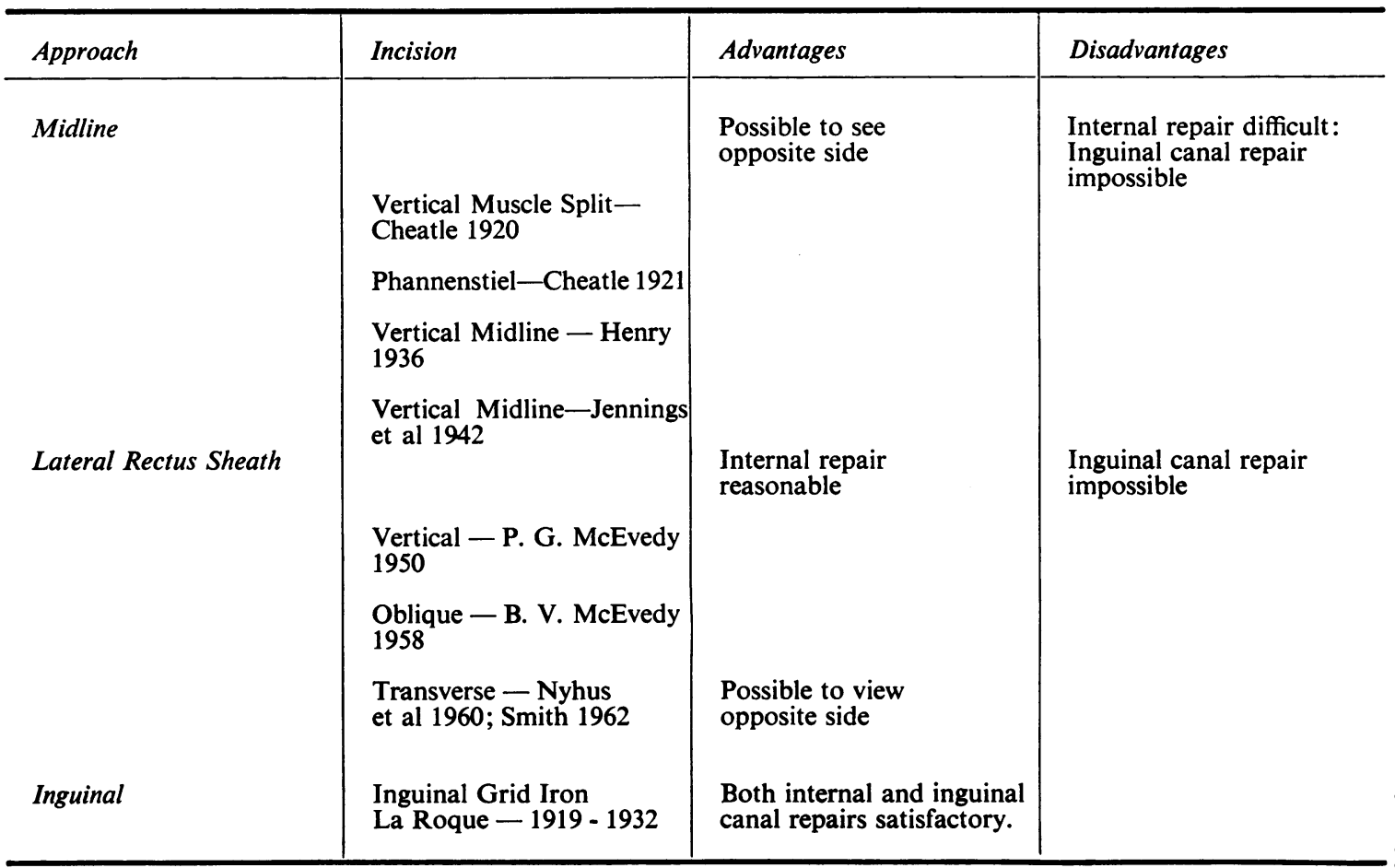

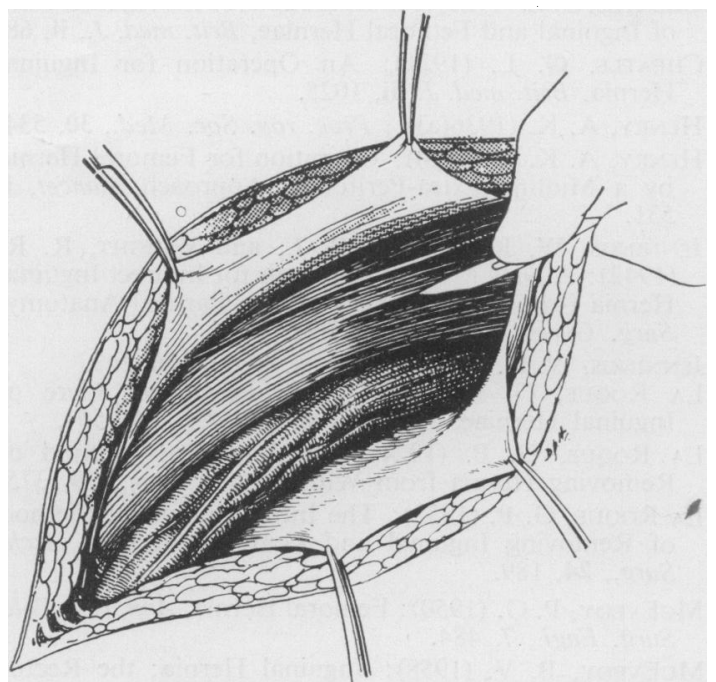

FIG. 1-The incision in internal oblique muscle above the internal ring.

The Internal Approach: Intraperitoneal

In trying to reach a synthesis of the best of these orthodox and heterodox approaches, it seems that a satisfactory compromise can be reached as follows:-

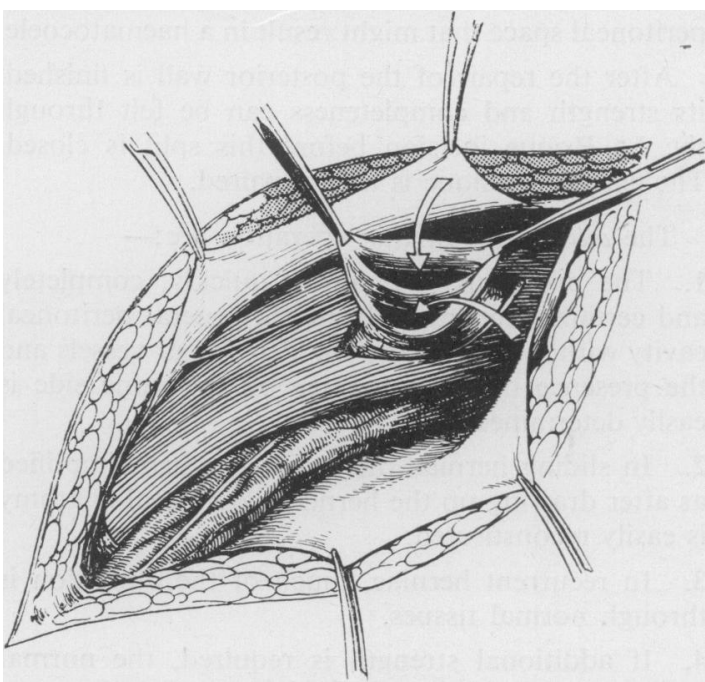

FIG. 2.-The peritoneum is divided across the watershed separating the hernial sac from the general peritoneal cavity.

After a routine inguinal incision, a $\mathrm{La}$ Roque muscle-split is made an inch above the internal ring and the peritoneum opened. (Fig. 1). The internal opening of the processus vaginalis can be seen and any other herniae will be apparent at this stage. 
The processus vaginalis is now divided well inside any thickening of the peritoneum at the internal ring (Fig. 2), while the bladder, vas and vessels, being visible, are easily avoided. Any minor procedures in the pelvis or on the appendix can be performed before the general peritoneal cavity is closed.

The internal ring and the posterior wall of the inguinal canal are now palpated and a decision made as to the repair required. If the patient is young, the posterior wall sound and the internal ring only slightly stretched, a sound repair with minimal damage to the inguinal canal shutter mechanism is best achieved by an internal repair only, drawing the mobilised vas and vessels medially and suturing the thickened transversalis fascial folds together laterally. If the defect is more marked and the posterior wall weak, probably a routine inguinal canal repair, such as a "Tanner slide," answers best; if the posterior wall is still weak a patch of fascia or mersalyn mesh can readily be stitched onto the inner aspect, where it is much more likely to be of value than one on the outer surface, where fixation is less secure.

The divided distal end of the processus vaginalis is left in situ, as damage is produced in removing it unless it slides out with minimal traction; this residual sac is not inflamed and does not lead to hydrocoele formation; it can be left open but is better closed if there is any oozing in the extraperitoneal space that might result in a haematocoele.

After the repair of the posterior wall is finished, its strength and completeness can be felt through the La Roque incision before this split is closed. The external oblique is then repaired.

The advantages of this operation are:-

1. The processus vaginalis is quickly, completely and certainly separated from the general peritoneal cavity without risk to the bladder, vas or vessels and the presence of other herniae on the same side is easily determined.

2. In sliding herniae the repair is much simplified as after drawing up the hernia, the normal anatomy is easily reconstituted.

3. In recurrent herniae, much of the operation is through normal tissues.

4. If additional strength is required, the normal repair can be supplemented by an internal repair, where patches of fascia or sheets of plastics can be more firmly fixed.

5. In strangulated herniae the inner aspect of the constricting ring is seen and resection is simple.

6. If the hernia is direct inguinal or femoral, it is still easily treated.

7. The appendix and pelvic organs can be treated if necessary, or an undescended testis mobilised $\underset{3}{3}$ much more adequately than through a simple $\stackrel{\mathbb{Q}}{\varrho}$ inguinal canal approach.

\section{Summary}

The extraperitoneal approach to inguinal herniae are discussed and the La Roque internal approach $\frac{\bar{\sigma}}{\bar{N}}$ is put forward as the operation of choice in all $\frac{\rho}{\sigma}$ inguinal herniae, with particular advantages in $\varnothing$ recurrent and sliding herniae and in those associated $\%$ with an undescended testis.

The operation is at least as simple as the routine inguinal canal operations and causes less damage $\vec{\omega}$ to the internal ring slide-and-shutter mechanism. In direct herniae, any defect filling patches can be applied onto the inner aspect of the posterior wall, where they can be fixed much more securely than $\hat{N}$ in the inguinal canal.

In routine use of this operation during the last three years there have been no complications from either the closed hernial sac remaining in situ or from the incision through the internal oblique muscle.

I am indebted to Mr. D. P. Hammersley of the $\frac{\mathbb{D}}{O}$ University of Newcastle upon Tyne Department of $\frac{\vec{D}}{\vec{D}}$ Surgery for the illustrations.

\section{REFERENCES}

Cheatle, G. L. (1920): An Operation for the Radical Curf of Inguinal and Femoral Herniae, Brit. med. J., ii, 68.

Cheatle, G. L. (1921): An Operation for Inguinal Hernia, Brit. med. J., ii, 1025.

Henry, A. K. (1936(a)):; Proc. roy. Soc. Med., 30, 534. HenRY, A. K. (1936(b)): Operation for Femoral Hernia by a Midline Extra-Peritoneal Approach, Lancet, i, 531.

Jennings, W. K., Anson, B. J., and Wright, R. R. (1942): A New Method of Repair for Indirect Inguinal Hernia Considered in Reference to Parietal Anatomy, Surg. Gynec. Obstet., 74, 697.

JenNINGS, W. K. (1960): Amer. J. Surg., 100, 243.

LA RoQue, G. P. (1919): The Permanent Cure of Inguinal Herniae, Surg. Gynec. Obstet., 29, 507.

LA RoQue, G. P. (1924): An Improved Method of Removing Hernia from Within, Ann. Surg., 79, 375.

LA RoQue, G. P. (1932): The Intra-Abdominal Method of Removing Inguinal and Femoral Hernia, Arch. Surg., 24, 189.

McEvedy, P. G. (1950): Femoral Hernia, Ann. roy. Coll. Surg. Engl., 7, 484.

McEvedY, B. V. (1958): Inguinal Hernia; the Rectus Sheath Approach, W.Afr. Med. J., 7, 106.

Nyhus, L. M., Condon, R. E., and Harkins, H. N. N (1960): Clinical Experience with Pre-Peritoneal N Hernial Repair of all Types of Hernia of the Groin, Amer. J. Surg., 100, 234.

Smith, A. N. (1962): A Rectus Sheath Extra-peritoneal Operation for Recurrent Inguinal Hernia, J. roy. Coll. Surg. Edinb., 7, 195.

Williams, C. (1938): The Advantages of the Abdominal Approach to Inguinal Hernia, Ann. Surg., 107, 917. 